\title{
DNA aberrations in urinary bladder cancer detected by flow cytometry and FISH
}

\begin{abstract}
Detection of molecular alterations is of potential significance for diagnosis and prognosis in bladder cancer. Fluorescence in situ hybridization (FISH) allows visualization and quantitation of genes and chromosomes on a cell by cell level and can easily be applied to urinary cells. To evaluate the sensitivity of FISH for detection of DNA aberrations in bladder cancer, formalin-fixed tissues of 293 tumors were examined by FISH and flow cytometry (FCM). Centromere probes for the chromosomes X, Y, 1, 7,9 , and 17 were used for FISH analysis. FISH was more sensitive for detection of quantitative DNA aberrations than FCM. An aberration of at least one chromosome was found in 107 of 108 tumors (99\%), which were tetraploid, aneuploid, or multiploid, and in 29 of 49 tumors (59\%), which were diploid, by FCM. The frequency of FISH aberrations showed greater differences between pTa $(47 \%)$ and p 11 tumors $(85 \% ; P<0.0001)$ than between stages pT1 and pT2-4 (98\%). The marked genetic difference between pTa and pT1 tumors argues against the concept of grouping pTa and pT1 tumors together as "superficial bladder cancer." The frequency of tumors with chromosomal aberrations detected by FISH increased with the number of chromosomes examined. Aneusomy was seen in $68 \%$ of grade 1 tumors examined for $\geq 4$ chromosomes, suggesting that the cytological diagnosis of bladder cancer recurrences could be substantially improved by FISH.
\end{abstract}

Key words Bladder neoplasms - Flow cytometry . Fluorescence in situ hybridization . Chromosome Y . Chromosome X . Chromosome 1 . Chromosome 7 . Chromosome 9 . Chromosome 17 . Aneuploidy

G. Sauter (四) - H. Moch - J. Richter · F. Jiang - R. Albrecht

H. Novotny - U. Wagner - L. Bubendorf - M.J. Mihatsch

Institute for Pathology, University of Basel,

Schönbeinstrasse 40, CH-4003 Basel, Switzerland

T. C. Gasser

Urologic Clinic, University of Basel,

Schönbeinstrasse 40, CH-4003 Basel, Switzerland

\section{Introduction}

Quantitative aberrations of the cellular DNA content occur frequently in bladder cancer [23]. Despite evidence for a diagnostic significance of DNA aneuploidy [5, 6, 24, 26], DNA measurement has not become a routine examination in bladder cancer patients. This is probably due to the need for expensive equipment and specially trained personel to perform either flow cytometry (FCM) or DNA image cytometry.

Fluorescence in situ hybridization (FISH) is an alternative method for detection of a disturbed cellular DNA content. FISH allows visualization and therefore quantitation of chromosomes and genes on a cell by cell basis $[9,10]$. FISH is technically easy and there is no need for specialized equipment. A major advantage of FISH as compared to total DNA measurement by FCM or image analysis is the capability to specifically examine chromosomes of interest for numerical aberrations. Since gains of chromosomes (polysomy) are frequently the result of a generalized alteration of the cellular DNA content involving most if not all chromosomes (tetraploidization), quantitation of a few chromosomes might be sufficient to detect DNA aneuploidy by FISH. Several studies have suggested that the sensitivity of FISH for detection of quantitative DNA aberrations exceeds the sensitivity of FCM $[8,9]$.

The aim of this study was to compare the sensitivity of FISH and FCM for detection of a disturbed DNA content in bladder cancer cells. In addition, the relationship between DNA aberrations and tumor grade and stage was examined.

\section{Materials and methods}

Biopsy material

Formalin-fixed paraffin-embedded tissue samples of primary transitional cell carcinomas of the urinary bladder were available from the archives of the Institute for Pathology, University of Basel, Switzer- 
land. A total of 293 tumors were selected for this study including 122 tumors that were previously examined by FISH for multiple chromosomal aberrations $[18,19,21]$. Tumor stage and grade were defined according to UICC [25] and WHO [14], using grades 1-3. Pure papillomas (grade 0 ) were excluded. Because of the limitations of transurethral biopsies in accurately determining the depth of invasion of higher stage bladder cancer, all tumors showing muscle invasion were categorized into one group (pT2-4). Ninety-six tumors were confined to the bladder mucosa (pTa), 67 showed minimal invasion limited to the lamina propria (pT1), and 130 were muscle-invasive (pT2-T4). Forty-five tumors were grade 1, 109 were grade 2, and 139 were grade 3 . Fifty-micrometer sections were taken from all tissue blocks. Nuclei were enzymatically dissociated as previously described [19].

\section{Flow cytometry}

FCM for DNA measurement was performed in 237 cases. Nuclei were stained with propidium iodide. Dissociated nuclei were analyzed with a FACScan flow cytometer (Becton-Dickinson Immunocytometry System, San Jose, CA). For each specimen 10000-20000 events were collected. The Multicycle Program (Phoenix Flow Systems, San Diego, CA) was used for data analysis. The lowest G0/G1 peak was considered diploid and given a DNA index (DI) of 1.00. The DI of other G0/G1 peaks was calculated as the ratio of their G0/G1 peak channel number of the diploid G0/G1 peak channel number of the histogram. Tumors with a nondiploid G0/G1 peak with a $\mathrm{DI} \leq 1.2$ were considered peridiploid. Tumors were defined as diploid if only one G0/G1 peak was present and the G2M peak was less than $15 \%$. A tumor was categorized as tetraploid if the DI was 1.9-2.1 or if the $\mathrm{G} 2 / \mathrm{M}$ peak of an otherwise diploid tumor was higher than or equal to $15 \%$. Tumors with $\mathrm{G} 0 / \mathrm{G} 1$ peaks not in the peridiploid (DI $\leq 1.2$ ) or tetraploid (DI 1.9-2.1) range were considered aneuploid. Multiploid tumors were defined as having more than one nondiploid G0/G1 peak. The coefficient of variation (CV) was calculated for each $\mathrm{G} 0 / \mathrm{G} 1$ peak. Only tumors with a diploid $\mathrm{CV} \leq 8 \%$ were included in this study.

Fluorescence in situ hybridization

FISH was performed as previously described $[19,21,22]$. The following centromere probes were used: $\mathrm{pY}$ (chr. Y), pBAMX (chr. X), pUC177 (chr. 1), p7alphaTET (chr. 7), pHUR98 (chr. 9), and p17H8 (chr. 17). All probes were generously supplied by the UCSF Resource for Molecular genetics (Dr. Joe W. Gray, Director). Probe labeling was performed with biotin-14-dATP or digoxigenin-11-dUTP by nick translation using standard protocols. For all hybridizations a dual-labeling procedure using centromere probes together with other centromeric or locus-specific cosmid probes was used as described [18-22]. Cells on slides were denatured in $70 \%$ formamide $/ 2 \mathrm{XSSC}$ (1X SSC is $0.15 \mathrm{M} \mathrm{NaCl}, 0.015 \mathrm{M} \mathrm{Na}$ citrate), $\mathrm{pH} 7$, at $75^{\circ} \mathrm{C}$ for $2.5 \mathrm{~min}$. Pretreatment of slides was with proteinase $\mathrm{K}(2.0 \mu \mathrm{g} / \mathrm{ml}$; Sigma) in PBS ( $\mathrm{pH} \mathrm{7.0)}$ ) for $7 \mathrm{~min}$ at $37^{\circ} \mathrm{C}$. The hybridization mixture was denatured for $5 \mathrm{~min}$ at $75^{\circ} \mathrm{C}$. For centromere/cosmid dual labeling, $10 \mu \mathrm{l}$ hybridization mixture consisted of $10 \mathrm{ng}$ cosmid probe, $10-30 \mathrm{ng}$ centromeric probe as well as $10 \mathrm{ng}$ unlabeled, sonicated (200-500 basepairs) human placental DNA (Sigma) in 50\% formamide, $10 \%$ dextran sulfate, and $2 X$ SSC (pH 7). For dual hybridizations using two centromere probes, the hybridization mixture was composed of 10-30 ng centromeric probe and $10 \mathrm{ng}$ unlabeled, sonicated ( $200-500$ basepairs), herring sperm DNA (Sigma) in $55 \%$ formamide, $10 \%$ dextran sulfate, and 2X SSC ( $\mathrm{pH} 7$ ). Hybridization was performed overnight at $37^{\circ} \mathrm{C}$. Lymphocyte metaphase spreads were used as controls to assure probe specificity. The slides were washed and immunostained in five steps as previously described [19]. If hybridization signals were weak, presumably due to low hybridization efficiency, hybridization was repeated using the same protocol with an increased proteinase $\mathrm{K}$ concentration (up to $10 \mu \mathrm{g} / \mathrm{ml}$ ). Proteinase $\mathrm{K}$ concentration was reduced if excessive nuclear damage was observed.

\section{Scoring of FISH signals}

Interpretation of centromere signals was as described $[21,22]$. In brief, only cells with at least one bright signal for one of the applied probes were scored to avoid false chromosomal losses due to insufficient hybridization efficiency. Slides were only scored if at least two-thirds of cells were interpretable. Small lymphocytes and granulocytes were disregarded. At least 100 nuclei were analyzed. Tumors were stratified according to fraction of polysomic cells (cells with > two centromere signals) and according to their predominant population $[19,22]$. A tumor was defined as polysomic for a chromosome if the fraction of polysomic cells was $\geq 10 \%$. A loss of the $Y$ chromosome was defined as the presence of $\geq 20 \%$ of nullisomic cells. The predominant population of a tumor was defined as the largest abnormal (aneusomic) population having $>20 \%$ of total cells [20]. A loss of chromosome 9 was defined if a tumor was either monosomic for chromosome 9 ( $\geq 20 \%$ monosomic cells) or its chromosome 9 copy number category (predominant population) was below the copy number category for all other chromosomes examined (except chromosome Y).

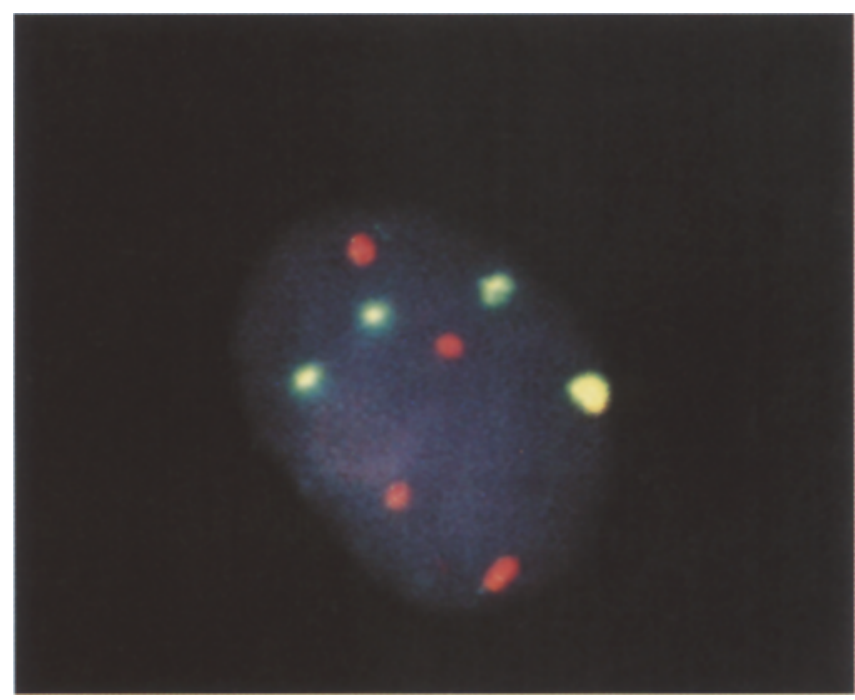

Fig. 1 Dual labeling FISH with probes for centromere 9 (red) and centromere 1 (green). Dissociated cell from a formalin-fixed bladder tumor containing four signals for both centromere 9 and centromere $1, \times 630$

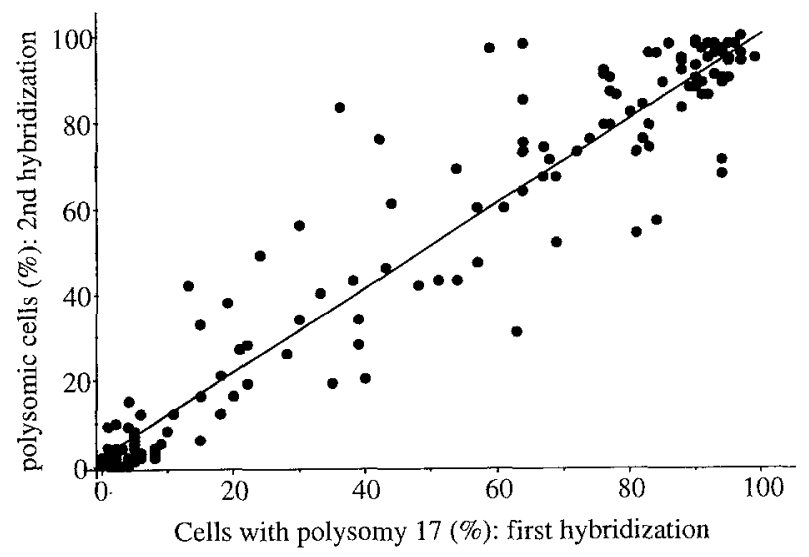

Fig. 2 Comparison of percentage of cells with polysomy 17 found in two separate hybridizations of 173 tumors. Each circle represents one tumor. $r^{2}=0.908, n=173, P<0.0001$ 


\section{Results}

Flow cytometry

The mean coefficient of variation $(\mathrm{CV})$ was 6.2 (range $2.8-18.1$ ) in all 237 tumors analyzed by FCM. Ploidy was determined in the $193(81 \%)$ cases with a diploid CV $\leq 8 \%$. Fifty-two of these tumors were diploid, 21 were peridi-

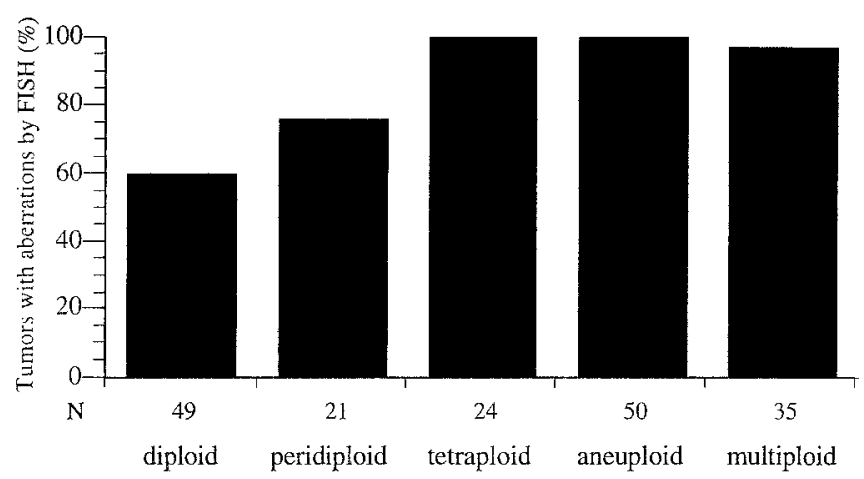

Fig. 3 FISH aberrations in tumors with differing ploidy type. Bars indicate the frequency in which polysomies or losses of at least one chromosome (X, Y, 1, 7, 9, 17) occurred in tumors with differing ploidy types ploid, 25 were tetraploid, 55 were aneuploid, and 40 were multiploid.

\section{FISH analysis}

FISH analyses with centromeric probes were successful in most cases. The slides were interpretable for centromere $\mathrm{X}$ in 184 of 191 (96\%), for centromere $\mathrm{Y}$ in 125 of 133 (94\%), for chromosome 1 in 127 of 135 (95\%), for chromosome 7 in 181 of 199 (91\%), for chromosome 9 in 186 of $215(87 \%)$, and for chromosome 17 in 238 of 280 tumors $(85 \%)$. Fifteen tumors were examined for one, 30 for two, 47 of three, 39 for four, 66 for five, and 56 for all six chromosomes. Most tumors were heterogeneous, containing more than one significant population with identical centromere counts. A cell of a representative case hybridized with probes for the centromeres 1 and 9 is shown in Fig. 1. The fraction of polysomic cells ranged between $0 \%$ and $100 \%$ for all chromosomes. A second hybridization for chromosome 17 was performed in 173 tumors. This showed that the fraction of polysomic cells was highly reproducible (Fig. 2). A polysomy ( $>2$ centromere signals in $\geq 10 \%$ of cells) was found for chromosome $X$ in $58 \%$, for chromosome $\mathrm{Y}$ in $40 \%$, for chromosome 1 in $76 \%$, for chromosome 7 in $68 \%$, for chromosome 9 in $52 \%$, and for chro-
Fig. 4 Polysomies in tumors with differing ploidy type. The frequency of polysomies of chromosomes $X, 1,7$, and 17 are shown as separate bars for tumors with differing ploidly types

Table 1 Predominant populations in bladder tumors examined by FISH

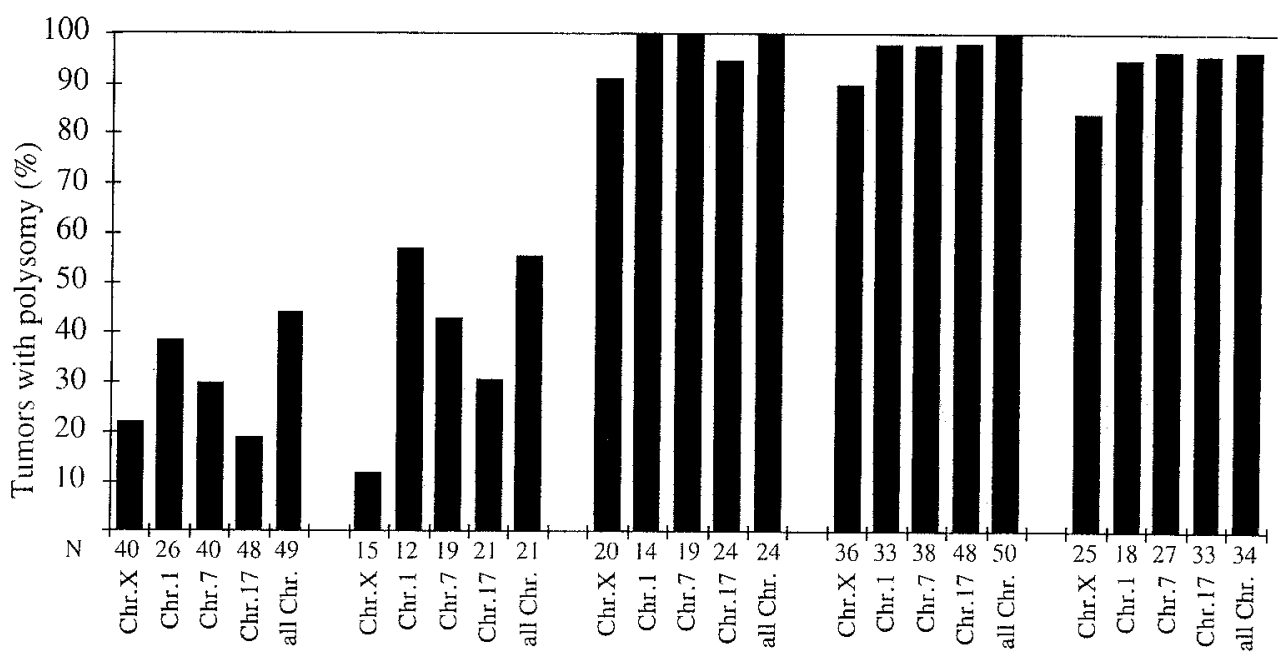

FCM: diploid peridiploid tetraploid aneuploid multiploid

\begin{tabular}{llrllllll}
\hline Chromosome & Sex & N & \multicolumn{7}{c}{ Predominant population (tumors \%) } \\
\cline { 3 - 8 } & & & 0 & 1 & 2 & 3 & 4 & $>4$ \\
\hline Chromosome Y & & 125 & $\underline{29}$ & $\mathbf{3 4}$ & 34 & 1 & 2 & 0 \\
Chromosome X & M & 131 & 0 & $\mathbf{4 5}$ & 51 & 3 & 1 & 0 \\
Chromosome 1 & F & 53 & 0 & $\underline{15}$ & $\mathbf{2 6}$ & 26 & 23 & 10 \\
Chromosome 7 & 127 & 0 & $\underline{2}$ & $\mathbf{2 7}$ & 33 & 33 & 5 \\
Chromosome 9 & 181 & 0 & $\underline{\underline{0.5}}$ & $\mathbf{3 2 . 5}$ & 22 & 38 & 7 \\
Chromosome 17 & 186 & 0 & $\underline{18}$ & $\mathbf{4 0}$ & 19 & 20 & 3 \\
\hline
\end{tabular}

${ }^{a}$ Largest aneusomic population having either $\geq 10 \%$ polysomic cells or $\geq 20 \%$ cells with less than the expected number of chromosomes

${ }^{b}$ The expected number of chromosomes is printed in bold for each chromosome. Tumors with chromosomal losses are underlined 


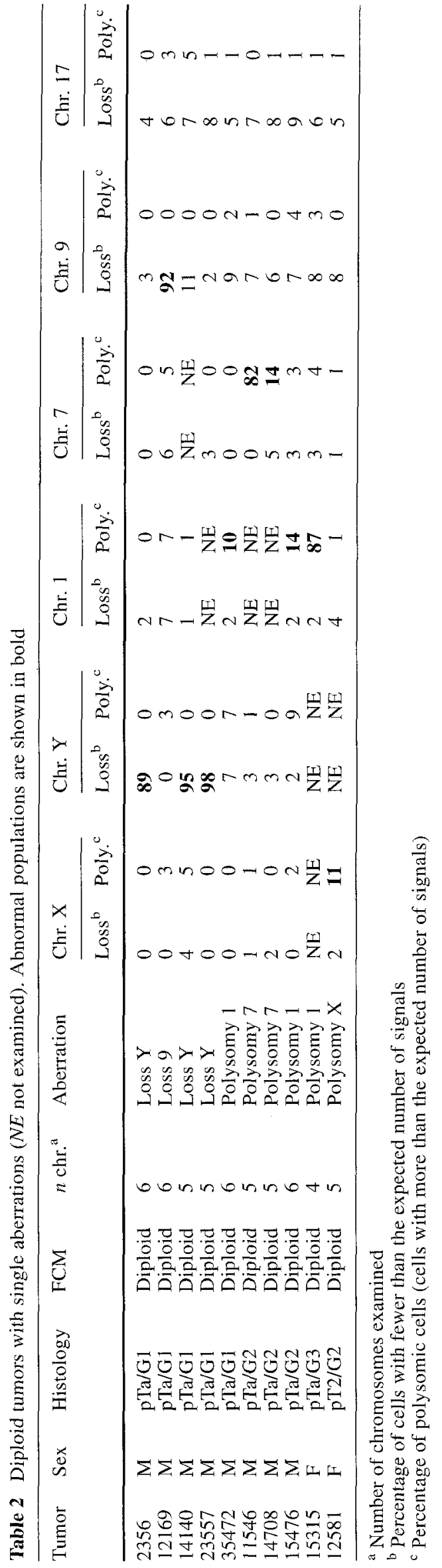

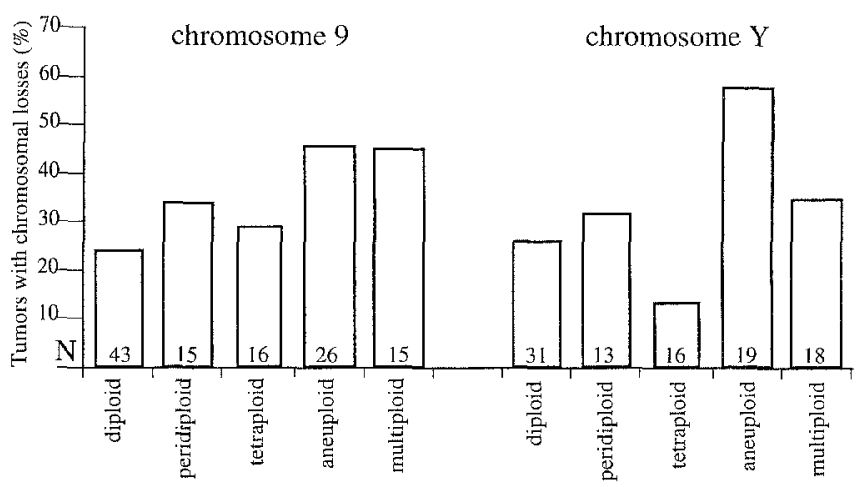

Fig. 5 Chromosomal losses and ploidy. The frequency of chromosome 9 and $\mathrm{Y}$ losses in tumors with different FCM findings is depicted as separate bars

mosome 17 in $64 \%$. A polysomy of at least one chromosome was seen in 191 of 273 tumors $(75.5 \%)$ examined by FISH. The distribution of the predominant populations for all chromosomes in all tumors is shown in Table 1. Fewer than the expected number of chromosomes were mostly found for chromosome Y ( $29 \%$ of tumors), and chromosome 9 (18\% of tumors). Interestingly there was a monosomy $X$ in $15 \%$ of the examined female patients while a nullisomy $X$ was never observed in male patients. Chromosomal losses were rare $(\leq 2 \%)$ for the chromosomes 1 , 7, and 17. A chromosome 9 loss according to our definition (including monosomy 9 and relative chromosome 9 losses) was found in $36 \%$ of tumors.

\section{FISH and FCM}

Chromosomal aberrations by FISH were found in 107 of 108 tumors that were tetraploid, aneuploid, or multiploid by FCM (Fig. 3). One multiploid tumor with a total of $37 \%$ aneuploid cells by FCM showed no chromosomal aberrations by FISH. However, only two chromosomes had been examined in this case. Numerical aberrations of at least one chromosome were also detected in 16 of 21 peridiploid (76\%), and 29 of 49 diploid tumors (59\%). A separate analysis of polysomies and chromosomal losses showed that polysomies were strongly associated with presence of tetraploid/aneuploid/multiploidy populations by FCM $(P<0.001$; Fig. 4$)$ while there was no significant relationship between losses of the chromosomes $Y$ and 9 and FCM ploidy (Fig. 5).

DNA aberrations and tumor phenotype

As a rule the fraction of tumors showing chromosomal aberrations increased with the number of FISH probes used (Fig. 6). This was prominent when one to four FISH probes were applied while there was no further increase in the number of polysomies detected when four to six probes were employed. Presence of a major DNA aberration by FCM (tetraploidy, aneuploidy, or multiploidy) and poly- 


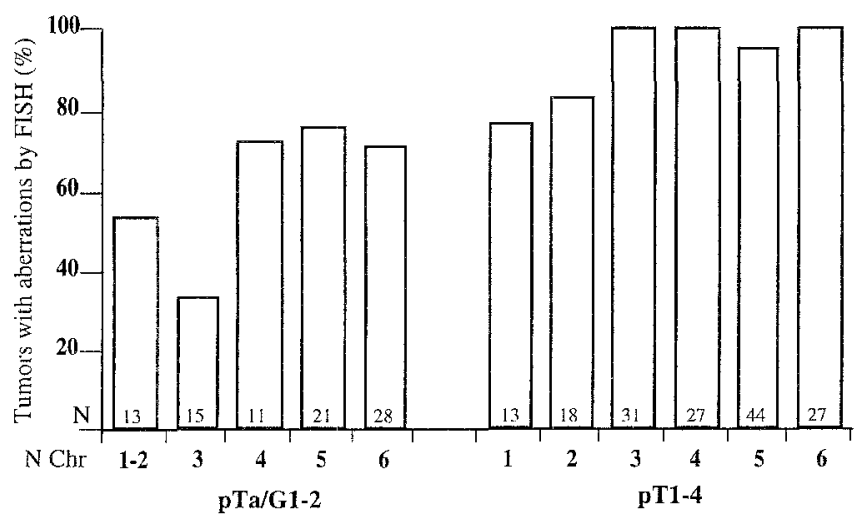

Fig. 6 Relationship between the frequency of aneusomy detection and the number of FISH probes applied. The percentage of tumors with detected polysomy or loss of at least one of the chromosomes $\mathrm{X}, \mathrm{Y}, 1,7,9$, and 17 as related to the number of chromosomes examined is shown separately for $\mathrm{pTa} / \mathrm{G} 1-2$ tumors (left) and invasive carcinomas $(p T 1-4) . N$ number of tumors examined, $N$ Chr number of chromosomes examined

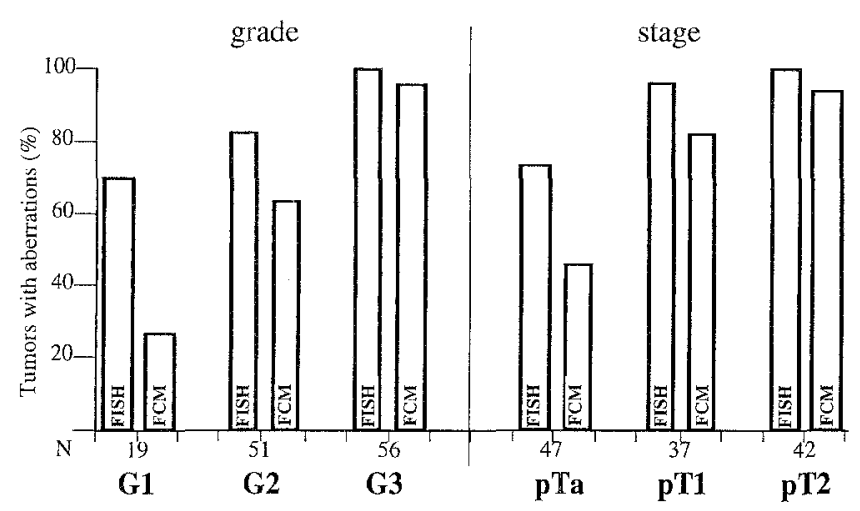

Fig. 7 Relationship of FISH and FCM findings with tumor grade and stage. The frequency of detected aberrations is shown as bars for each grade and stage. Tumors with less than four chromosomes examined by FISH were excluded from this analysis

somies as detected by FISH were strongly associated with a high tumor grade and an invasive tumor stage (pT1-4). The fraction of detectable aberrations increased continuously from grade 1 over grade 2 to grade 3 tumors with highly significant differences between grade 1 and grade 2 tumors $(P<0.0001)$ and between grade 2 and grade 3 tumors $(P<0.0001$, Fig. 7$)$. A marked difference was also seen between pTa and pT 1 carcinomas $(P<0.0001)$, while there was no significant further increase in the number of aberrations from $\mathrm{pT} 1$ to $\mathrm{pT} 2-4$ carcinomas. In contrast to polysomies, losses of the chromosomes $\mathrm{Y}$ and 9 were not significantly associated with tumor grade and stage (data not shown).

\section{Discussion}

These results show that alterations of the cellular DNA content occur frequently in bladder carcinomas. This is consistent with a number of previous studies using image cytometry, flow cytometry, or FISH for detection of DNA aberrations $[1,8,9,18,23]$. Importantly, the differences in aneuploidy and polysomies of all chromosomes examined were clearly more prominent between $\mathrm{pTa}$ and $\mathrm{pT} 1$ tumors than between pT1 and pT2-4 tumors. The concept that pT1 tumors are biologically closer to pT2-T4 tumors is also supported by marked differences in p53 lesions and other cytogenetic alterations between $\mathrm{pTa}$ and $\mathrm{pT} 1$ carcinomas $[19,20]$. These results argue against the current concept of grouping pTa and pT1 tumors together as "superficial bladder carcinomas."

Three of the FISH probes used in this study were selected because alterations of these chromosomes are known to occur frequently in bladder tumors. Chromosome 9 carries one or several tumor suppressor genes with relevance for bladder cancer, loss of chromosome $Y$ has been suggested to have prognostic significance in bladder tumors, and for chromosome 7 it was suggested that a trisomy could represent an initial event in bladder carcinogenesis $[11,16,17]$. The chromosomes $\mathrm{X}, 1$, and 17 were selected because high-quality centromere probes were available.

Our FISH analyses of multiple chromosomes in individual tumors showed that polysomies were usually not restricted to one chromosome but involved several if not all chromosomes examined. This suggests that most chromosomal aberrations which are detectable by FISH are nonspecific, being the result of an event involving more or less the entire genome. This fits well with models suggesting that progression of malignant tumors goes along with sequential changes of their cellular DNA content. It is believed that early genomic alteration includes losses or gains of a few chromosomes or subchromosomal regions that can result in a peridiploid DNA histogram [4]. Development of aneuploidy is likely to occur through tetraploidization of previously diploid or peridiploid tumors and a subsequent loss of chromosomal material [23]. Our hypothesis that most polysomies detected by FISH may be the result of a previous tetraploidization is further supported by the equally high frequencies of polysomies of all chromosomes examined in tumors with an FCM result consistent with a previous tetraploidization (tetraploid, aneuploid, and multiploid tumors) as shown in Fig. 4.

To evaluate whether numerical aberrations of the chromosomes examined can be specific events potentially involved in the initiation of bladder cancer, we screened for tumors having only one chromosomal aberration. Such tumors are likely to be diploid by FCM, since the sensitivity of FCM is not sufficient to allow detection of gains or losses of single chromosomes in formalin-fixed tissues. Among 126 tumors which were examined for at least 4 chromosomes by FISH and also by FCM, there were only 10 diploid tumors having a numerical alteration of a single chromosome (Table 2). While it cannot be excluded that these ten tumors had aberrations of other chromosomes not examined in this study, this result shows that sole numerical aberrations of the selected chromosomes are rare in bladder cancer. Five different aberrations were found 
among ten tumors having only one aberration and no single chromosome was solely involved in more than three tumors. Therefore, an important role of numerical aberration of the examined chromosomes for bladder cancer initiation seems unlikely. This does not rule out, however, that an altered function of genes on these chromosomes for example MTS $1 / 2$ on chromosome 9 - is important for bladder cancer development.

The comparison of FISH and FCM findings revealed a superior sensitivity of FISH for detection of DNA aberrations. The finding of chromosomal aberrations by FISH in all but one of 108 tumors found to be tetraploid, aneuploid, or multiploid by FCM is comparable to the results of previous studies on prostate, bladder, and ovarian carcinomas, where the fraction of FCM aneuploid tumors without detectable aberrations by FISH was low [7]. The finding of chromosomal aberrations in 59\% of the FCM diploid tumors is also in agreement with a previous study compairing FISH and FCM in bladder cancer [7]. Interestingly the fraction of tumors with aberrations was only slightly higher in peridiploid than in diploid tumors. It is possible that the application of additional probes could have resulted in a better result in the subset of peridiploid tumors, even though the application of more than four probes did not further improve the sensitivity of FISH in the entire patient set.

There was no association between chromosome $\mathrm{Y}$ and 9 losses and FCM aneuploidy in this set of patients. This observation is consistent with two different types of "genetic instability" in bladder cancer. One type of instability obviously allows tetraploidization and development of aneuploidy. An inactivation of the p53 tumor suppressor gene is likely to be involved in the pathway leading to this type of instability since p53 alterations are strongly linked to polysomies in bladder cancer [19,21,22]. It is estimated that mutations or deletions of $\mathrm{p} 53$ occur in more than $50 \%$ of invasive bladder tumors $[2,3,13,15,27]$. The conspicuous lack of an association between chromosomal losses and FCM aneuploidy raises the possibility that the development of minor chromosomal aberrations including chromosome $\mathrm{Y}$ or 9 losses is facilitated by a different type of "genetic instability." Such a second mechanism could be independent of $\mathrm{p} 53$ alterations since losses of the chromosomes $\mathrm{Y}$ and 9 were not related to p53 immunostaining in previous studies $[21,22]$.

A major problem in the management of bladder cancer patients is the diagnosis of recurrent bladder tumors. Although urinary cells are readily accessible for cytological examination, the sensitivity (and specificity) of cytology alone is poor in low-grade tumors [12]. Previous studies have suggested that DNA measurement of urinary cells by FCM or image cytometry could be a valuable tool for the diagnosis of bladder cancer recurrences (reviewed in [26]). The results of this study indicate that the sensitivity of FISH for detection of aberrant urinary cells could be even higher than the sensitivity of FCM. Importantly the sensitivity of FISH is particularly high in grade 1 tumors, where the sensitivity of urinary cytology ranges between $16 \%$ and $30 \%$. In this study DNA aberrations were detected by FCM in $26 \%$ and by FISH in $68 \%$ of grade 1 tumors. Considering that not all FISH probes applied in this study were selected for chromosomes or loci known to be frequently affected in low-grade bladder tumors, it appears likely that FISH detection of aberrant urinary cells could be further improved by an optimized set of probes. If so, FISH could be a powerful tool for urinary tract cytology, even though its specificity will not reach $100 \%$ because of rare chromosomal aberrations in normal urothelium [22].

In summary, these data show that an aberrant DNA content is frequent in bladder carcinomas. The striking difference in DNA aberrations between pTa and pT1 tumors challenges the concept of grouping these tumors together as "superficial bladder carcinomas." The high sensitivity of FISH for detection of DNA alterations in grade 1 tumors suggests that the cytological diagnosis of bladder cancer recurrences could be substantially improved by FISH. This is especially true because FISH is a simple method requiring very little material with the potential for routine application.

Acknowledgements The authors thank Ms. Carole Egenter, Ms Martina Storz, and the staff of the Pathology Department, University of Basel, for their technical assistance. The work was supported by Schweizerische Krebsliga, Basler Krebsliga, Krebsforschung Schweiz, and Stiftung für klinische Krebsforschung Basel.

\section{References}

1. Blomjous EC, Schipper NW, Baak JP, Vos W, De Voogt HJ, Meijer CJ (1989) The value of morphometry and DNA flow cytometry in addition to classic prognosticators in superficial urinary bladder carcinoma. Am J Clin Pathol 91:243

2. Dalbagni G, Presti J, Reuter V, Zhang Z-F, Fair W, Sarkis A, Cordon-Cardo C (1993) Molecular genetic alterations of chromosome 17 and p53 nuclear overexpression in human bladder cancer. Diagn Mol Pathol 2:4

3. Esrig D, Spruck CHI, Nichols PW, Chaiwun B, Steven K, Groshen S, Chen SC, Skinner DG, Jones PA, Cote RJ (1993) p53 Nuclear protein accumulation correlates with mutations in the p53 gene, tumor grade, and stage in bladder cancer. Am J Pathol $143: 1389$

4. Giaretti W (1994) A model of DNA aneuploidization and evolution in colorectal cancer. Lab Invest 71:904

5. Gustafson H, Tribukait B (1985) Characterization of bladder carcinomas by flow DNA analysis. Eur Urol 11:410

6. Gustafson H, Tribukait B, Esposti $\mathrm{P}$ (1982) DNA profile and tumor progression in patients with superficial bladder cancer. Urol Res 10:13

7. Hopman A, Ramaekers F, Raap A, Beck J, Devilee P, van der Ploeg M, Vooijs G (1988) In situ hybridization as a tool to study numerical chromosome aberrations in solid bladder tumors. Histochemistry $89: 307$

8. Hopman A, Poddighe P, Smeets A, Moesker O, Beck J, Vooijs $G$, Ramaekers F (1989) Detection of numerical chromosome aberrations in bladder cancer by in situ hybridization. Am J Pathol 135:1105

9. Hopman A, Moesker O, Smeets A, Pauwels R, Vooijs G, Ramaekers F (1991) Numerical chromosome 1, 7, 9, and 11 aberrations in bladder cancer detected by in situ hybridization. Cancer Res 51:644

10. Kallioniemi O, Kallioniemi A, Kurisu W, Thor A, Chen L, Smith H, Waldman F, Pinkel D, Gray J (1992) C-erB-2 oncogene amplification in breast cancer analyzed by fluorescence in situ hybridization. Proc Natl Acad Sci USA 89:5321 
11. Kamb A, Gruis N, Weaver-Feldhaus J, Liu Q, Harshman K, Tavtigian S, Stockert E, Day III R, Johnson B, Skolnick M (1994) A cell cycle regulator potentially involved in genesis of many tumor types. Science 264:436

12. Koss L, Deitch D, Ramanathan R, Sherman A (1985) Diagnostic value of cytology of voided urine. Acta Cytol 29:810

13. Miyamoto H, Kubota Y, Shuin T, Torigoe S, Hosaka M, Iwasaki Y, Danenberg K, Danenberg PV (1993) Analyses of p53 gene mutations in primary human bladder cancer. Oncol Res 5:6

14. Mostofi $F$ (1973) Histological typing of urinary bladder tumors. World Health Organization, Geneva

15. Olumi A, Tsai Y, Nichols P, Skinner D, Cain D, Bender L, Jones P (1990) Allelic loss of chromosome 17p distinguishes high grade from low grade transitional cell carcinomas of the bladder. Cancer Res 50:7081

16. Ruppert J, Tokino T, Sidransky D (1993) Evidence for two bladder cancer suppressor loci on human chromosome 9. Cancer Res 53:5093

17. Sandberg A, Berger C (1994) Review of chromosome studies in urological tumors. II. Cytogenetics and molecular genetics of bladder cancer. J Urol151:545

18. Sauter G, Moch H, Moore D, Carroll P, Kerschmann R, Chew K, Mihatsch M, Gudat F, Waldman F (1993) Heterogeneity of erbB-2 gene amplification in bladder cancer. Cancer Res 53: 2199

19. Sauter G, Deng G, Moch H,Kerschmann R, Matsumura K, DeVries S, George T, Fuentes J, Carroll P, Mihatsch M, Wald- man F (1994) Physical deletion of the 553 gene in bladder cancer: Detection by fluorescence in situ hybridization. Am J Pathol 144:756

20. Sauter G, Carroll P, Moch H, Kallioniemi A, Kurschmann R, Narayam P, Mihatsch M, Waldman F (1995) Increased c-myc gene copy number in advanced bladder cancer. Am $\mathbf{J}$ Pathol 146:1131

21. Sauter G, Moch H, Carroll P, Kurschmann R, Mihatsch M, Waldman F (1995) Chromosome 9 loss detected by fluorescence in situ hybridization. Int J Cancer 64:99

22. Sauter G, Moch H, Wagner U, Novotna H, Gasser T, Mattarelli G, Mihatsch M, Waldman F (1995) Y Chromosome loss detected by FISH in bladder cancer. Cancer Gen Cytogen 82:163

23. Tribukait B (1987) Flow cytometry in assessing the clinical aggressiveness of genitourinary neoplasms. World $\mathbf{J}$ Urol 5:108

24. Tribukait B, Gustafson H, Esposti P (1982) The significance of ploidy and proliferation in the clinical evaluation of bladder tumors. Br J Urol 54:130

25. UICC (1978) TNM classification of malignant tumours. International Union against Cancer, Geneva

26. Wheeless LL, Badalament RA, DeVere WRW, Fradet Y, Tribukait B (1993) Consensus review of the clinical utility of DNA cytometry in bladder cancer. Cytometry $14: 478$

27. Williamson MP, Elder PA, Knowles MA (1994) The spectrum of TP53 mutations in bladder carcinoma. Genes Chromosom Cancer 9:108 